Research Article

\title{
Cutaneous Lesions in Iranian Neonates and Their Relationships with Maternal-Neonatal Factors: A Prospective Cross-Sectional Study
}

\author{
Hossein Firouzi, ${ }^{1}$ Iman Jalalimehr, ${ }^{1}$ Zahra Ostadi, $^{2}$ and Siavash Rahimi ${ }^{1}$ \\ ${ }^{1}$ Department of Pediatrics, Ramsar Campus, Mazandaran University of Medical Sciences, Sari, Iran \\ ${ }^{2}$ Department of Pediatrics, Shahid Rajaee Hospital, Mazandaran University of Medical Sciences, Tonekabon, Iran
}

Correspondence should be addressed to Siavash Rahimi; siavash.rahimi@hotmail.com

Received 11 February 2020; Accepted 20 April 2020; Published 5 May 2020

Academic Editor: Giuseppe Stinco

Copyright (c) 2020 Hossein Firouzi et al. This is an open access article distributed under the Creative Commons Attribution License, which permits unrestricted use, distribution, and reproduction in any medium, provided the original work is properly cited.

\begin{abstract}
Cutaneous lesions are common in the neonatal period and mostly physiological, transient, and self-limited; uncommonly, they are pathological and require treatment and cooperation between neonatologists and dermatologists. Particular conditions, like prematurity, can influence the onset, type, and evolution of cutaneous manifestations. Of the several articles in the literature about skin findings in newborns, only a few were performed in Iran. We aimed to investigate dermatological findings in a sample of neonates within the first three days of life and to evaluate the association between skin lesions and neonatal- or maternal-related variables. A total of 1202 newborns, hospitalized in the Department of Pediatrics of Imam Sajjad Hospital of Ramsar and Shahid Rajaee Hospital of Tonekabon, Iran, for two years, were examined. All skin findings were recorded, and information on neonatal and maternal variables was collected and analyzed to detect statistically significant associations. Skin lesions were present in 958 newborns (79.8\%). The prevalence of milia, erythema toxicum, salmon patch, and Mongolian spots were $45.2 \%, 43 \%, 37.3 \%$, and $37 \%$, respectively. Natural vaginal delivery, use of medication, term gestation, and maternal disease were associated with a higher incidence of cutaneous lesions in neonates. Milia, erythema toxicum, Mongolian spots, and genital hyperpigmentation were seen more frequently in the male gender. Conversely, skin desquamation was seen more frequently in females. Among maternal diseases, gestational diabetes mellitus, urinary tract infection, preeclampsia, hypertension, psychiatric disorders, and uterine infection were associated with a higher prevalence of cutaneous lesions. Neonatal cutaneous lesions are a common source of concern in parents and inexperienced physicians. Therefore, prompt recognition of neonatal cutaneous lesions is essential in order to avoid unnecessary diagnostic and therapeutic procedures.
\end{abstract}

\section{Introduction}

The skin of the infant differs from that of the adult, in that it is thinner and delicate, has weaker intercellular attachments, and produces less sweat and sebaceous gland secretions and it is more susceptible to several infections. The neonatal period is defined as the first 28 days of extrauterine life. The neonatal integumentary system offers physical protection, fluid balance regulation, immunosurveillance, and heat preservation, therefore playing a vital role in the newborn's transition from an aqueous to an air-dominant environment $[1,2]$. In this period, dermatological findings are prevalent and can differ significantly from physiological and selflimited lesions caused by the developmental process of the integumentary system to severe pathological issues, which requires interventions and collaboration between specialists [3]. Therefore, early recognition is essential to differentiate benign lesions from more serious disorders and to provide proper counseling to parents. Several fetal, maternal, and environmental factors, such as prematurity, congenital infections, ethnicity, and maternal drug use, can influence the onset, type, and evolution of cutaneous lesions [3-6]. Previous studies have been performed regarding cutaneous lesions in neonates and their association with fetal 
conditions and maternal factors, yet only a few of these studies were performed in a diverse population of Iran [7-9]. Our study aimed to investigate dermatological findings in a sample of Iranian neonates in the north of Iran within the first 72 hours of their extrauterine life and to evaluate the potential influence of several neonatal-, parental-, or pregnancy-related variables in the onset of dermatological findings.

\section{Materials and Methods}

This multicenter hospital-based study was conducted at two major general hospitals in the west of Mazandaran province, Iran. In this cross-sectional study, 1202 consecutive newborns were evaluated for two years (2017-2019) at Imam Sajjad hospital of Ramsar and Shahid Rajaee hospital of Tonekabon, Mazandaran University of Medical Sciences. All newborns in regular postnatal wards who were no more than 72 hours of age were included. The study protocol was approved by the ethical committee of Mazandaran University of Medical Sciences before enrolling any participants. Also, prior to enrollment, written informed consent was obtained from participants' parents. A single dermatologist and neonatologist examined all patients within 72 hours of life. Entire skin surface, including palms, soles, genitalia, and scalp, was carefully examined, and cutaneous lesions were diagnosed based on clinical and morphologic features; no skin biopsy was performed. In the event of diagnoses requiring observation or therapy, further management was shared with the parents. Data regarding the neonatal history of the participating neonates (gestational age, maternal comorbidities, mode of delivery, birth weight, maternal age, parity, and maternal drug use) were obtained from the official medical profiles and interviews by a neonatologist.

Based on 35\% prevalence, the confidence level of $99 \%$, the margin of error $5 \%$, and population size of 100,000 samples were calculated to be 1202 with the following equations:

$$
\begin{aligned}
& X=\frac{Z_{(\alpha / 2)}^{2} * p * q}{\mathrm{MOE}^{2}} \\
& n=2\left(N * \frac{X}{X+N-1}\right) .
\end{aligned}
$$

At the end of the study, the collected data were analyzed using SPSS v22.0 (IBM Corp., Armonk, N.Y, USA). Descriptive statistical methods-means, standard deviations (SDs), and frequencies-were used to analyze the variables. Comparisons of variables were performed using the chisquare test, Fischer exact test, and independent $t$-test. $P$ values of less than 0.05 were considered to indicate statistical significance.

\section{Results}

In this study, 1200 newborns were evaluated. Due to missing documents, 2 patients were excluded. Maternal and neonatal characteristics and their association with cutaneous lesions are mentioned in Tables 1 and 2, respectively. 958 (79.8\%) of newborns had cutaneous lesions. The prevalence of different maternal diseases and their association with cutaneous lesion are shown in Table 3. The prevalence of cutaneous lesions based on genders is presented in Table 4.

\section{Discussion}

Although the majority of cutaneous conditions in the newborns are benign, they are the most encountered condition in general neonatal pediatric practice [10]. Thus, it is essential to differentiate physiologic skin lesions from pathologic ones to avoid unnecessary diagnostic or therapeutic procedures and parents' concerns. This study was a clinical and statistical survey of neonatal cutaneous conditions during the first three days of extrauterine life in Iranian neonates. In this study, $79.8 \%$ of newborns had at least one cutaneous lesion. The four most common cutaneous conditions found in this study were milia (45.2\%), erythema toxicum (43\%), salmon patch (37.3\%), and Mongolian spots (37\%).

There are few reports from Iran, which are overviewed here. Karegar Maher et al. reported salmon patch and Mongolian spots frequency in the Northwest of Iran [7]. According to the results, the frequency rates of Mongolian spots and salmon patch among 1000 newborns were found to be $32.3 \%$ and $14.5 \%$, respectively. Maternal age was the only factor that showed a statistically significant association with the salmon patch. In addition, upper eyelid and sacral regions were found to be the most common site of the salmon patch and Mongolian spots, respectively [7].

Shajari et al. evaluated the incidence of different birthmarks in 503 neonates in Tehran, Iran [9]. Epstein's pearls, Mongolian spots, erythema toxicum, sucking blisters, salmon patch, milia, petechial, and mottling were found in $88.27 \%, 81 \%, 54 \%, 52.1 \%, 52 \%, 46 \%, 0.08 \%$, and $0.06 \%$ of neonates, respectively. Petechia was reported more frequently in vaginal delivery and neonates with higher birth weight. Mottling was more common in premature and lowbirth-weight babies [9].

Khoshnevisasl et al. evaluated the incidence of birthmarks in neonates born in Zanjan, Iran [8]. Among examined 500 neonates, $95.6 \%$ had at least one lesion. The most common skin lesions were Epstein's pearl (60.4\%) and Mongolian spots (56\%), respectively. Mongolian spots were significantly associated with gestational age and black hair color. Epstein's pearl was significantly associated with NVD; on the other hand, erythema toxicum was significantly associated with $\mathrm{C} / \mathrm{S}$. There was a significant relationship between milia and increasing gestational age [8].

The prevalence of milia in previous studies has ranged from 1.4 to $93.1 \%$ [11-15]. In our study, it was found in 542 (45.2\%) of neonates, which is similar to the previous report from Iran $[8,9]$. The association of milia with genders has been conflicting across studies. Significant association of male gender has been reported in by Zagne and Fernandes [15] and Behera et al. [16]. On the other hand, Gokdemir et al. [12] and Jain et al. [17] reported a significant association of female gender with milia.

Erythema toxicum was noted in $43 \%$ of our population, which is conflicting with both Iranian studies $[8,9]$. However, non-Iranian studies reported a lower incidence of 
TABLE 1: Maternal characteristics and their association with cutaneous lesions.

\begin{tabular}{lcc}
\hline Maternal factors & Number $(\%)$ & Association with skin lesions $(P$ value $)$ \\
\hline Maternal age, mean \pm SD & $26.7 \pm 6.3$ & $>0.05$ \\
17-24 years & $480(40)$ & \\
25 to 32 years & $522(43.5)$ & $>0.05$ \\
33 to 40 years & $198(16.5)$ & \\
Number of pregnancies & & \\
1 & $864(72)$ & $<0.01$ \\
2 & $240(20)$ & \\
3 & $72(6)$ & $<0.01$ \\
$>3$ & $24(2)$ & $<0.01$ \\
Mode of delivery & & \\
NVD & $960(80)$ & \\
C/S & $240(20)$ & \\
Use of medication during pregnancy & $240(20 \%)$ & \\
Maternal disease & $314(26.3)$ & \\
\hline
\end{tabular}

NVD, natural vaginal delivery. C/S, caesarian section.

TABLE 2: Neonatal characteristics and their association with cutaneous lesions.

\begin{tabular}{lccc}
\hline Neonatal factors & Number & Percentage & Association with cutaneous lesions $(P$ value $)$ \\
\hline Gender (male) & 610 & 50.9 & 0.758 \\
Gestational age & & & $<0.01$ \\
$\quad$ Term & 1058 & 88.2 & 0.476 \\
$\quad$ Preterm & 142 & 11.8 & \\
Birth weight & 120 & 10 & \\
$\quad<2500$ gr & 1080 & 90 & \\
$\quad>2500$ gr & & & \\
\hline
\end{tabular}

TABle 3: Type of maternal disease and their association with cutaneous lesions.

\begin{tabular}{lccc}
\hline Type of maternal disease & Number & Percentage & Association with cutaneous lesions $(P$ value $)$ \\
\hline Gestational diabetes mellitus (GDM) & 92 & 7.7 & $<0.01$ \\
Urinary tract infection (UTI) & 60 & 5 & $<0.01$ \\
Preeclampsia & 42 & 3.5 & $<0.05$ \\
Hypertension & 34 & 2.8 & $<0.05$ \\
Psychiatric disorders & 12 & 1 & $<0.01$ \\
Uterine infections & 12 & 1 & $<0.01$ \\
Hypothyroidism & 12 & 1 & $>0.05$ \\
Thrombocytopenia & 8 & 0.66 & $>0.05$ \\
Placental abnormalities & 6 & 0.5 & $>0.05$ \\
Asthma & 6 & 0.5 & $>0.05$ \\
Thalassemia & 6 & 0.5 & $>0.05$ \\
Anemia & 6 & 0.5 & $>0.05$ \\
Other diseases & 18 & 1.5 & $>0.05$ \\
Total & 314 & 26.3 & $<0.01$ \\
\hline
\end{tabular}

erythema toxicum $[4,18]$. Positive correlation with $\mathrm{C} / \mathrm{S}$ and term gestation was reported in previous studies. In our study, it was shown to be significantly more common in males, which is similar to earlier reports from Behera et al. [16].

Salmon patch was the third common cutaneous lesion in our study, with an incidence of $37.3 \%$, which was in the range of 14.5 to $52 \%$ reported in previous Iranian studies [7-9].

Mongolian spots are bluish grey macules that evolve in consequence of the residual melanocytes trapped in the dermis in the course of embryonal migration. It affected $37 \%$ of our neonates' population that was lower than previous studies in Iran, India, and Brazil [4, 8, 9, 19]. Earlier, Ferahbas et al. [13] and Khoshnevisasl et al. [8] both suggested that the Mongolian spots are related to gestational age and hair color but no association with sex and mode of delivery. Our findings suggested a significant association of Mongolian spots with the male gender.

Skin desquamation was observed in $21.1 \%$ of our population, which is relatively similar to the study in Brazil [18]. In contrast to Behera et al., our study found a significant 
TABLE 4: The frequency of cutaneous lesions seen in neonates in the first 72 hours of extrauterine life and their distribution according to gender $(n)(\%)$.

\begin{tabular}{lcccc}
\hline Skin lesion & Total number $(n=958)$ & Male $(n=490)$ & Female $(n=468)$ & Association with cutaneous lesions $(P$ value $)$ \\
\hline Milia & $542(45.2)$ & $306(25.5)$ & $236(19.7)$ & 0.0004 \\
Erythema toxicum & $516(43)$ & $282(23.5)$ & $234(19.5)$ & 0.02 \\
Salmon patch & $448(37.3)$ & $240(20)$ & $208(17.3)$ & 0.17 \\
Mongolian spots & $444(37)$ & $252(21)$ & $192(16)$ & 0.001 \\
Skin desquamation & $254(21.1)$ & $112(9.3)$ & $142(11.8)$ & 0.015 \\
Genital hyperpigmentation & $54(4.5)$ & $50(4.2)$ & $4(0.3)$ & $<0.01$ \\
Hemangiomas & $22(1.8)$ & $6(1)$ & $5(0.8)$ & 0.06 \\
Pigmented nevus & $12(1)$ & $4(0.33)$ & $8(0.66)$ & 0.25 \\
Miliaria & $8(0.66)$ & $4(0.33)$ & $4(0.33)$ & 1 \\
Pustular melanosis & $6(0.5)$ & $4(0.33)$ & $2(0.16)$ & 0.68 \\
Total number of lesions & 2311 & 1260 & 1035 & \\
\hline
\end{tabular}

association with female gender and incidence of skin desquamation [16].

Genital hyperpigmentation was the fifth prevalent dermatological finding in our study and was observed in 50 of the male population and 4 of our females with a significantly higher frequency rate of $4.2 \%$ and $0.3 \%$, respectively.

We also assessed the associations between neonatalmaternal factors and prevalence and type of cutaneous lesions in neonates during 72 hours after their delivery. Our data suggest that there were no significant correlation between neither maternal age nor the number of pregnancies with an incidence of cutaneous lesions. Interestingly, in the study conducted in the northwest of Iran, maternal age was the only factor that showed a statistically significant association with salmon patch. In another study conducted in Messina, Italy, the skin lesion frequency was significantly higher in those born to primigravida mothers $[7,20]$. There was a higher rate of cutaneous lesions among neonates who were born through NVD in comparison with those with C/S in the present study. Mechanical trauma during delivery and higher gestational age among NVD neonates can be the reason for this difference.

This is the first study in the north of Iran to assess associations between dermatological findings and maternalneonatal factors. The advantage of our study was focusing on maternal-neonatal factors in detail in addition to frequency rates of lesions, while other similar studies in Iran had discussed only prevalence. However, our data are limited to two hospitals located in the west of Mazandaran province. Therefore, we recommend further studies in different hospitals. Also, in our study, we did not measure the association of neonatal-maternal factors with location and type of cutaneous lesions; this matter can be addressed in future studies.

\section{Conclusion}

To the best of our knowledge, our study is the first that investigates the incidence of skin lesions during the first three days of life in neonates from the north of Iran. In this study, $79.8 \%$ of newborns had at least one cutaneous lesion. The prevalence of milia, erythema toxicum, salmon patch, and Mongolian spots were 45.2\%, 43\%, 37.3\%, and 37\%, respectively. NVD, use of medication, term gestation, and maternal disease were associated with a higher incidence of cutaneous lesions in neonates. Cutaneous lesions are known to be affected by maternal hormones, especially androgens [4]. Consequently, with a longer gestational period, the fetus is more exposed to maternal hormones and more cutaneous lesions develop. Milia, erythema toxicum, Mongolian spots, and genital hyperpigmentation were seen more frequently in the male gender. Conversely, skin desquamation was seen more frequently in females. Among maternal diseases, GDM, UTI, preeclampsia, hypertension, psychiatric disorders, and uterine infection were associated with higher skin lesions. These disorders or their treatments can alter hormonal balance during gestation, resulting in a higher prevalence of neonatal cutaneous lesions. On the other hand, UTI and uterine disease are known to affect neonatal skin through direct infection [4]. From a clinical point of view, it must be emphasized that cutaneous findings are common in newborns and are often a source of parental anxiety and medical concern for inexperienced clinicians. For these reasons, comprehensive knowledge of neonatal cutaneous lesions is crucial for clinicians to avoid unnecessary (and, in some cases, potentially harmful) diagnostic and therapeutic measures. This is important particularly in the circumstances, like low-birth-weight and premature neonates, where medical conditions necessitate special care and iatrogenic complications after treatment are common. This study highlights the high prevalence of different cutaneous lesions in the Iranian population and its associated factors in order to deliver a better understanding of neonatal cutaneous lesions in clinical practice.

\section{Data Availability}

The data used to support the findings of this study are available from the corresponding author upon request.

\section{Ethical Approval}

The study protocol was approved by the ethical committee of Mazandaran University of Medical Sciences before enrolling any participants. Also, prior to enrollment, written informed consent was obtained from participants' parents. 


\section{Conflicts of Interest}

The authors declare that there are no conflicts of interest.

\section{Authors' Contributions}

HF conceptualized and supervised the project. IJ, SR, and $\mathrm{ZO}$ collected the data. HF analyzed the data. SR prepared the draft. ZO, HF, and IJ revised the drafted manuscript. All authors have read and approved the manuscript.

\section{Acknowledgments}

This study was part of the doctorate dissertation of Iman Jalalimehr and funded by Mazandaran University of Medical Sciences' Vice-Chancellor of research.

\section{References}

[1] A. Chadha and M. Jahnke, "Common neonatal rashes," $\mathrm{Pe}$ diatric Annals, vol. 48, no. 1, pp. e16-e22, 2019.

[2] B. Z. Rayala and D. S. Morrell, "Common skin conditions in children: neonatal skin lesions," FP Essent, vol. 453, pp. 11-17, 2017.

[3] N. R. O'Connor, M. R. McLaughlin, and P. Ham, "Newborn skin: part I. Common rashes," American Family Physician, vol. 77, no. 77, pp. 47-52, 2008.

[4] F. T. Haveri and A. C. Inamadar, "A cross-sectional prospective study of cutaneous lesions in newborn," ISRN Dermatology, vol. 2014, Article ID 360590, 8 pages, 2014.

[5] M. Kahana, M. Feldman, Z. Abudi, and S. Yurman, "The incidence of birthmarks in Israeli neonates," International Journal of Dermatology, vol. 34, no. 10, pp. 704-706, 1995.

[6] L. Techasatian, V. Sanaphay, P. Paopongsawan, and L. A. Schachner, "Neonatal birthmarks: a prospective survey in 1000 neonates," Global Pediatric Health, vol. 6, Article ID 2333794X1983566, 2019.

[7] M. H. Karegar Maher, S. Heidar Abady, and A. Tabrizi, "Salmon patch and Mongolian spot frequency in the northwest of Iran: a descriptive study," Iranian Journal of Neonatology, vol. 7, no. 3, pp. 24-28, 2016.

[8] P. Khoshnevisasl, M. Sadeghzadeh, S. Mazloomzadeh, and A. Zanjani, "The incidence of birthmarks in neonates born in Zanjan, Iran," Journal of Clinical Neonatology, vol. 4, no. 1, pp. 8-12, 2015.

[9] H. Shajari, A. Shajari, and N. S. M. Habiby, "The incidence of birthmarks in Iranian neonates," Acta Medica Iranica, vol. 45, no. 5, pp. 424-426, 2007.

[10] N. Carmona, M. G. Bentz, M. M. Bhagwat, and M. D. Cabana, "Newborn skin findings in the nursery," Clinical Pediatrics, vol. 57, no. 7, pp. 850-853, 2018.

[11] A. A. El-Moneim and R. E. El-Dawela, "Survey of skin disorders in newborns: clinical observation in an Egyptian medical centre nursery," Eastern Mediterranean Health Journal, vol. 18, no. 1, pp. 49-55, 2012.

[12] G. Gokdemir, H. Erdogan, A. Koslu, and B. Baksu, "Cutaneous lesions in Turkish neonates born in a teaching hospital," Indian Journal of Dermatology, Venereology and Leprology, vol. 75, no. 6, p. 638, 2009.

[13] A. Ferahbas, S. Utas, M. Akcakus, T. Gunes, and S. Mistik, "Prevalence of cutaneous findings in hospitalized neonates: a prospective observational study," Pediatric Dermatology, vol. 26, no. 2, pp. 139-142, 2009.
[14] T. Agung Senapathi, I. Budiarta, and M. Astuti, "Low dose spinal anaesthesia for cesarean section in gravida with rheumatic heart disease," Indian Journal of Dermatology, Venereology, and Leprology, vol. 57, no. 1, pp. 25-28, 1991.

[15] V. Zagne and N. Fernandes, "Dermatoses in the first $72 \mathrm{~h}$ of life: a clinical and statistical survey," Indian Journal of Dermatology, Venereology, and Leprology, vol. 77, no. 4, pp. 470-476, 2011.

[16] B. Behera, Y. Kavadya, P. Mohanty, D. Routray, S. Ghosh, and L. Das, "Study of physiological and pathological skin changes in neonates: an east Indian perspective," Indian Journal of Paediatric Dermatology, vol. 19, no. 1, pp. 40-47, 2018.

[17] N. Jain, B. Rathore, A. Agarwal, and A. Bhardwaj, "Cutaneous lesions in neonates admitted in a tertiary setup neonatal intensive care unit," Indian Journal of Paediatric Dermatology, vol. 14, no. 3, pp. 62-66, 2013.

[18] F. P. Reginatto, D. DeVilla, F. M. Muller et al., "Prevalence and characterization of neonatal skin disorders in the first $72 \mathrm{~h}$ of life," Jornal de Pediatria (Versão em Português), vol. 93, no. 3, pp. 238-245, 2017.

[19] J. R. P. D. Almeida, M. M. D. A. Alchorne, and M. A. Rozman, "Incidence of skin conditions in neonates born at a public hospital associated with some variables in pregnant women at risk," Einstein (São Paulo), vol. 8, no. 2, pp. 143-148, 2010.

[20] R. Giuffrida, F. Borgia, L. De Pasquale, F. Guarneri, C. Cacace, and S. P. Cannavò, "Skin lesions in preterm and term newborns from Southern Italy and their relationship to neonatal, parental and pregnancy-related variables," Giornale Italiano di Dermatologia e Venereologia: Organo Ufficiale, vol. 154, no. 4, pp. 400-404, 2019. 